VIII ${ }^{\text {èmes }}$ Journées Nationales Génie Civil - Génie Côtier, Compiègne, 7-9 septembre 2004

\title{
Liquéfaction, affaiblissement et rupture d'un lit de vase sous l'action de la houle
}

\author{
Ricardo Silva Jacinto \\ Chercheur, Ifremer - Centre de Brest, Direction de l'Environnement et de l'Aménagement Littoral, \\ B.P. 70, 29280 Plouzané. 02.98.22.46.61. \\ Ricardo.Silva.Jacinto@ifremer.fr.
}

\section{Résumé}

L'érosion des sédiments cohésifs sous l'action des vagues a un caractère soudain : elle correspond à une disparition des couches de vase en consolidation et, souvent, à un arrachement et dispersion sous forme de galets mous des couches de vase déjà consolidées. Dans cette étude, un modèle est présenté afin d'analyser l'interaction entre les vagues et les fonds vaseux. Pour des conditions d'agitations données, en fonction de la profondeur et des caractéristiques rhéologiques du sédiment, cette approche permet d'estimer la profondeur de rupture et l'épaisseur de la couche sédimentaire mobilisable. Puis, il est possible d'estimer l'amplitude d'oscillation de l'interface et d'en déduire la déformation du sédiment qui, de pair avec la densité, détermine les propriétés rhéologiques et l'état de liquéfaction.

\section{$\underline{\text { Abstract }}$}

The erosion of cohesive sediments under wave action is a sudden process. Both consolidating layers, which are liquefied and entrained, and consolidated layers can be completely eroded in few hours. A modelling approach for assessing rheological changes of mud beds under wave action is proposed. For a given wave, water depth and sediment rheology, the model provides the thickness of the dynamic sediment layer, the shear strain in the bed and the final rheological and liquefaction state.

\section{Introduction}

Dans beaucoup d'estuaires et d'autres zones côtières, les fonds sont constitués majoritairement de sédiments fins cohésifs dits vaseux. Ces zones côtières sont naturellement abritées des agitations provenant du large et de l'océan ouvert. Cependant, les vagues (houle et clapot) peuvent y être présentes lors d'évènements extrêmes et contribuer à une érosion importante et soudaine des fonds sédimentaires (Silva Jacinto et Le Hir, 2001). Cette contribution peut être déterminante de l'équilibre morpho-sédimentaire de ces zones côtières (Waeles et al., 2002). L'érosion des fonds vaseux sous l'action des vagues est un phénomène complexe. Les vagues peuvent générer des contraintes au sein de la couche sédimentaire et y induire un mouvement susceptible de devenir instable (De Wit, 1995). Ce mouvement altère les propriétés mécaniques de la couche sédimentaire, diminue sa rigidité et augmente son érodabilité. Dans certaines situations, le fond vaseux peut se liquéfier sous l'action des vagues (Lindenberg et al., 1989 ; Feng, 1992 ; De Wit, 1995) et être ainsi rapidement entraîné par les courants ou couler sous l'action de la gravité si la pente du fond le permet. En outre, la mise en mouvement de la couche sédimentaire peut avoir un effet sur la propagation des vagues, en dissipant son énergie et en modifiant leur dispersion (Yamamoto et al., 1986). L’objectif de ce travail est de proposer une synthèse de ces effets et un modèle susceptible d'être intégré dans les outils de modélisation hydrodynamique et de transport 3D à caractère opérationnel. 
Depuis le modèle présenté par Gade (1958), plusieurs modèles analytiques de l'interaction entre les vagues et les fonds sédimentaires montrent que le moteur du mouvement engendré au sein de la couche est lié au gradients horizontaux de pression et non au frottement à l'interface. Dans les modèles analytiques développés, le comportement rhéologique de la couche sédimentaire est supposé $a$ priori et rarement validé par des mesures rhéométriques du comportement de vases naturelles. Le comportement de la couche sédimentaire a été ainsi supposé comme purement visqueux (Dalrymple et Liu, 1978 ; Ng Co, 2000), purement élastique (Mallard et Dalrymple, 1977), viscoélastique (MacPherson, 1980 ; Maa et Mehta, 1988) ou viscoplastique (Mei et Liu, 1987). Dû à leur caractère linéaire, ces modèles analytiques concernent davantage la réponse du fond, liquéfié ou pas, que la mise en liquéfaction et l'altération rhéologique qui l'accompagne.

Sur la base d'un critère de seuil, Foda et al. (1993) ont proposé un modèle non linéaire pour une couche sédimentaire faiblement stratifiée permettant la quantification de la profondeur de liquéfaction. Cette profondeur étant définie comme l’interface entre une couche sédimentaire à comportement newtonien et une couche inférieur dont le comportement demeure viscoélastique linéaire. Cette approche ne reproduit pas les processus d'érosion de masse observés sous l'action des vagues. En effet, le processus de liquéfaction et de rupture ont lieu au sein du sédiment près des interfaces avec des couches plus profondes et plus rigides (Silva Jacinto et Le Hir, 2001). Le sédiment pouvant alors garder une certaine cohérence structurelle près de l'interface entre l'eau et le sédiment. Sous l'effet des souspressions des vagues, des copeaux de vase plus ou moins importants peuvent alors être arrachés du fond sous la forme de galets mous. Des expériences menées en canal par Yamamoto et al. (1986) avaient déjà mis en évidence que la dissipation visqueuse au sein de la couche sédimentaire ne dépend seulement pas des gradients de pression induits mais aussi du gradient vertical des propriétés mécaniques (i.e. rigidité) au sein du sédiment : la stratification du lit favorise la dissipation visqueuse.

\section{Rhéologie des sédiments cohésifs}

Les caractéristiques viscoélastiques des matériaux cohésifs sont souvent déduites par rhéométrie rotative : e.g. Chou et al. (1993) pour une vase modèle ; Silva Jacinto et Le Hir (2001) et Aubry et al. (2003) pour des vases estuariennes naturelles. L'échantillon est soumis à une déformation $(\gamma)$ uniforme entre deux plans. Cette déformation est imposée en fonction du temps $(\mathrm{t})$ de manière sinusoïdale avec une fréquence $\omega$ et une amplitude $\gamma_{0}$ :

$$
\gamma=\gamma_{0} \cdot \mathrm{e}^{-\mathrm{i} \omega \mathrm{t}}
$$

La contrainte de cisaillement résultante est approché par une sinusoïdale :

$$
\tau=\tau_{0} \cdot \mathrm{e}^{-\mathrm{i}(\omega \mathrm{t}+\delta)}
$$

où $\tau$ décrit la contrainte de cisaillement, $\tau_{0}$ l'amplitude de cette contrainte et $\delta$, désigné angle de perte, quantifie le déphasage entre les deux signaux. Ce déphasage impose l'existence d'un module de cisaillement complexe :

$$
\begin{aligned}
& \mathrm{G} \equiv \frac{\tau}{\gamma}=\mathrm{G}_{0} \cdot \mathrm{e}^{-\mathrm{i} \delta} \text { où } \mathrm{G}_{0}=\frac{\tau_{0}}{\gamma_{0}} \\
& \mathrm{G}=\mathrm{G}^{\prime}-\mathrm{i} \mathrm{G}^{\prime \prime}=\mathrm{G}_{0}(\cos \delta-\mathrm{i} \cdot \sin \delta)
\end{aligned}
$$

La partie réelle de $G$ est le module de conservation G' qui représente la partie élastique de la réponse. La partie imaginaire de $G$ correspond au module de perte G" qui représente la 
dissipation visqueuse. Le paramètre $\mathrm{G}_{0}$ est nommé module complexe. Quand le module de cisaillement complexe est un réel $(\delta=0)$, la réponse est purement élastique.

Quand l'angle de perte approche $\pi / 2$, le module de cisaillement complexe devient un imaginaire pur et la réponse est purement visqueuse. De manière équivalente, le comportement du sédiment peut être décrit par une viscosité cinématique complexe (v) :

$$
v=\tau /(\rho \dot{\gamma})=\left(G^{\prime \prime}+i G^{\prime}\right) / \rho \omega
$$

où $\rho$ est la masse volumique (ci-après densité) du sédiment et $\dot{\gamma}$ la dérivée temporelle de la déformation. Le concept de viscosité complexe est très utile car il permet de résoudre les équations de la mécanique dans l'espace complexe à l'aide du formalisme de la mécanique des fluides.

Les paramètres viscoélastiques des sédiments cohésifs augmentent de quelques ordres de grandeur avec la densité des sédiments et diminuent de quelques ordres de grandeur avec l'amplitude de la déformation (e.g. Aubry et al., 2003). Ces deux effets peuvent être clairement séparés. Les mesures rhéométriques montrent qu'à très faible déformation $\left(\gamma_{0}=\gamma_{\varepsilon}=\right.$ $\left.10^{-3}\right)$, le module complexe à faible déformation $\left(G_{0, \varepsilon}\right)$ augmente de manière exponentielle avec la densité du sédiment :

$$
G_{0, \varepsilon}=\alpha \cdot e^{\beta \frac{\rho-\rho_{e}}{\rho_{e}}}
$$

où $\rho_{\mathrm{e}}$ est la densité de l'eau, prise comme référence $\left(1000 \mathrm{~kg} \cdot \mathrm{m}^{-3}\right)$, et $\alpha$ et $\beta$ sont des paramètres propres au matériau. Prenant comme exemple d'application la vase de la Vasière Nord de l'estuaire de la Seine, les mesures rhéométriques obtenues pour six densités différentes (figure 1) peuvent être approchées par le modèle exprimé par l'équation (6) pour des valeurs de $\alpha$ et $\beta$ respectivement de $10 \mathrm{~Pa}$ et 15. Pour la vase de l'estuaire de la Penzé, Aubry et al. (2003) ont obtenu le même type de relation ( $\alpha=0.5$ Pa et $\beta=20)$. Ces auteurs ont observé la même relation de type exponentielle (même $\beta$ ) vis à vis de la densité pour d'autres paramètres rhéologiques tels que la viscosité apparente et la contrainte seuil obtenues par des tests de fluage. Chou et al. (1993) obtiennent une relation du même type pour la contrainte seuil obtenue en rotation $(\beta=17.8)$.

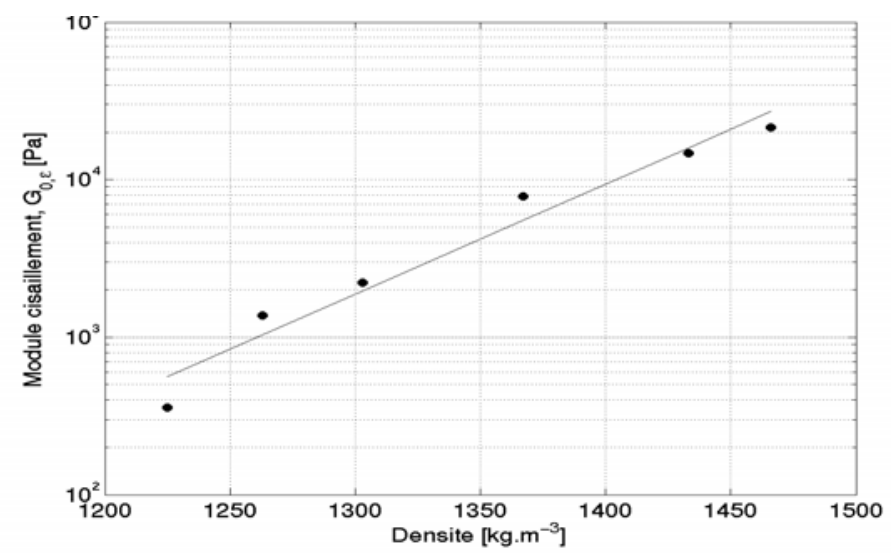

Figure 1 - Module complexe à faible déformation en fonction de la densité (vase de Seine). Comparaison du modèle proposé (trait) aux mesures rhéométriques. 
Pour des amplitudes de déformation plus importantes, le module complexe diminue relativement à sa valeur à faible déformation. Cette diminution relative est indépendante de la densité. On peut en effet écrire pour une densité et une amplitude de déformation données :

$\mathrm{G}_{0}\left(\rho, \gamma_{0}\right)=\phi\left(\gamma_{0}\right) \cdot \mathrm{G}_{0, \varepsilon}(\rho)$

L'exemple de la vase de Seine, présenté sur la figure 2 (gauche), illustre l'effet déstructurant de l'amplitude de déformation et l'indépendance de cet effet vis à vis de la densité.

Le modèle proposé s’exprime ainsi de la façon suivante :

$$
\begin{array}{ll}
\phi=1 & \text { si } \gamma<6.10^{-3} \\
\phi(\gamma)=0.003 . \gamma^{-1.14} & \text { si } 6.10^{-3} \leq \gamma<1 \\
\phi=0.003 & \text { si } \gamma \geq 1
\end{array}
$$

Le rapport entre les modules de perte et de conservation est donné par le facteur de perte (tan $\delta)$. L'angle de perte est lui aussi essentiellement déterminé par l'amplitude de déformation. Pour la vase de Seine, d'après les résultats expérimentaux, nous pouvons définir trois zones de comportements différentiés (figure 2). Tout d'abord un domaine de faible déformation $\left(\gamma<6.10^{-3}\right)$ où l'angle de perte est relativement constant. Puis, une zone de transition $\left(6.10^{-3}<\gamma<1\right)$ où l'angle de perte augmente de manière logarithmique avec l'amplitude de déformation. Et enfin, une zone de grande déformation où la vase est liquéfiée car l'angle de perte acquière une valeur constante et proche de $\pi / 2$. Le modèle choisi s'écrit :

$$
\begin{cases}\delta(\gamma)=0.2 & \text { si } \gamma<6.10^{-3} \\ \delta(\gamma)=0.24 \cdot \ln (\gamma)+1.45 & \text { si } 6.10^{-3} \leq \gamma<1 \\ \delta(\gamma)=1.45 & \text { si } \gamma \geq 1\end{cases}
$$
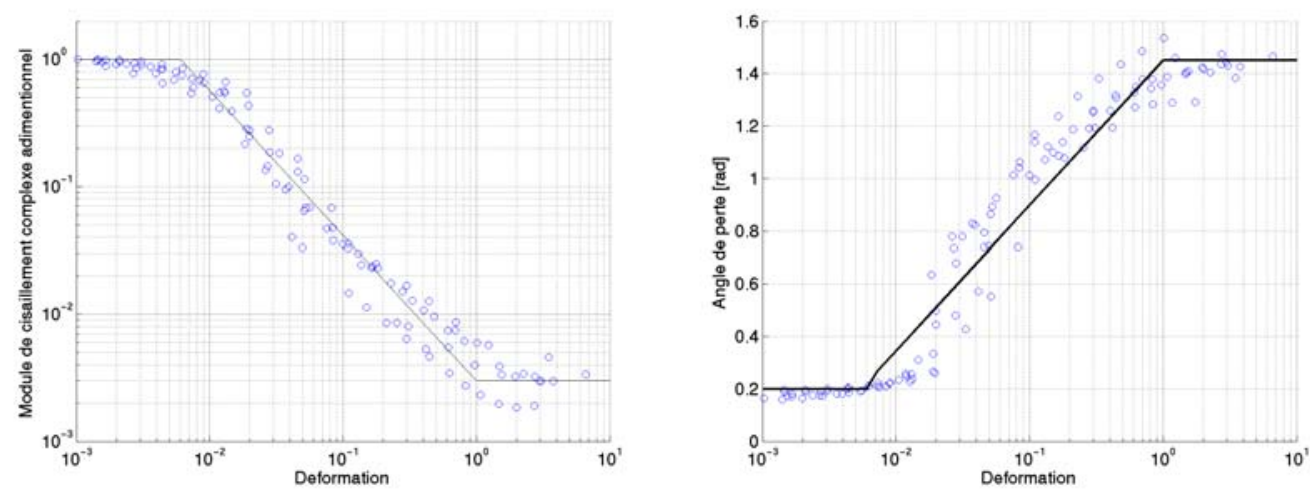

Figure 2 - Gauche : Evolution relative ( $\phi$ ) du module complexe par rapport aux faibles deformations pour l'ensemble des densités et tests réalisés sur de la vase de Seine et modèle adopté (trait). Droite : Evolution de l'angle de perte en fonction de l'amplitude de déformation pour l'ensemble des densités et tests. 
En conclusion, la réponse viscoélastique rhéologique des vases naturelles soumises à des mouvements oscillatoires est caractérisé par une plage quasi-linéaire où la cohésion induit un comportement majoritairement élastique. Au delà d'une certaine déformation critique, indépendante de la densité, les paramètres rhéologiques diminuent de quelques ordres de grandeur. Pour de larges déformations, la perte de cohésion se traduit par une augmentation de l'angle de perte : le mode dissipatif devient dominant et le matériau s'approche d'un comportement visqueux. Nous appelons liquéfaction cette altération de comportement.

\section{$\underline{\text { 3.Profondeur de liquéfaction }}$}

Si l'épaisseur de la couche de sédiment mobilisable est inférieure à la couche limite de houle au sein du sédiment avant liquéfaction (1 à $5 \mathrm{~m}$ ), la distribution des pression est hydrostatique et, du fait des petites déformations, la distribution des contraintes se traduit par un équilibre entre le gradient horizontal de pression et le gradient vertical de la contrainte de cisaillement (MacPherson, 1980). Pour des ondes sinusoïdales, l'amplitude de la contrainte de cisaillement au sein du sédiment augmente ainsi linéairement avec la profondeur :

$$
\tau_{0}=\mathrm{G}_{0, \varepsilon} \cdot \gamma=\mathrm{kp}_{0} \cdot(\mathrm{d}-\mathrm{z})
$$

où $\mathrm{k}$ est le nombre d'onde, $\mathrm{p}_{0}$ l'amplitude des oscillations de pression à l'interface, $\mathrm{d}$ l'épaisseur de la couche sédimentaire et z la coordonnée verticale au sein de la couche sédimentaire et supposée positive vers le haut. Les surfaces de rupture ou de liquéfaction $\left(\mathrm{z}=\mathrm{Z}_{\mathrm{liq}}\right)$ correspondront à des niveaux où la déformation est maximale (où la déformation critique sera atteinte). Ce niveau de liquéfaction correspond ainsi à des niveau où la dérivée verticale de la déformation passe par un zéro :

$$
\left.\frac{\partial \gamma}{\partial z}\right|_{\mathrm{Z}_{\text {liq }}}=-\frac{\mathrm{kp}_{0}}{\mathrm{G}_{0, \varepsilon}^{2}}\left(\mathrm{G}_{0, \varepsilon}+\frac{\mathrm{dG}_{0, \varepsilon}}{\mathrm{dz}}\left(\mathrm{d}-\mathrm{z}_{\text {liq }}\right)\right)=0 \Leftrightarrow\left(\mathrm{z}_{\text {liq }}-\mathrm{d}\right)=\frac{\mathrm{G}_{0, \varepsilon}}{\mathrm{dG}_{0, \varepsilon} / \mathrm{dz}}
$$

Si l'occurrence de rupture ou liquéfaction dépend du forçage et du niveau de déformation induit, la surface de liquéfaction ou de rupture dépend des seules propriétés rhéologiques de la couche sédimentaire. La distance de cette surface de rupture à l'interface eau-sédiment est donnée par l'échelle de variation verticale du module complexe. Ainsi, la surface de rupture d'une couche très stratifiée sera proche de l'interface ; celle d'une couche homogène sera proche de la limite inférieure de la couche sédimentaire, à l'interface avec des couches inférieures plus rigides.

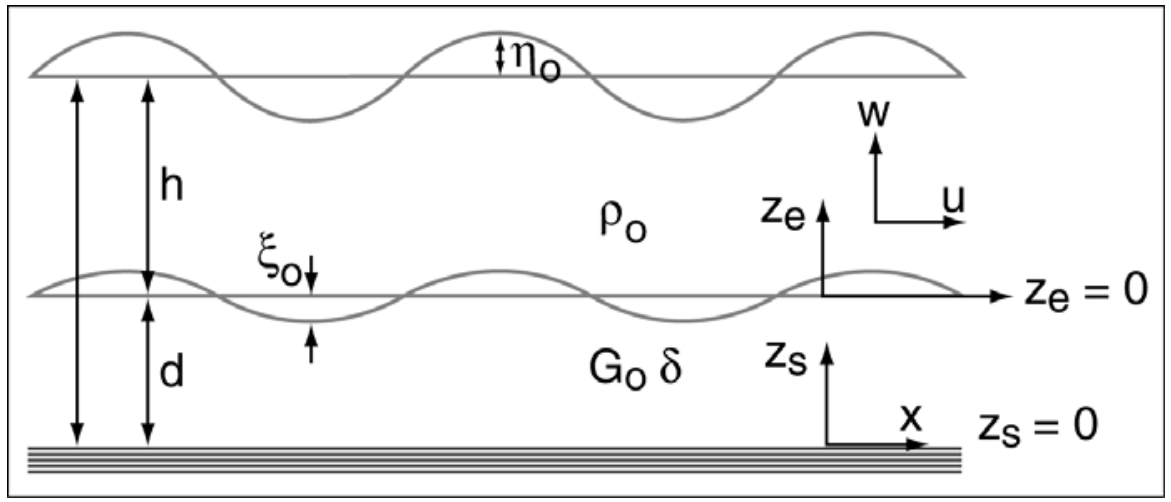

Figure 3 - Schéma de définition du problème à deux couches. 


\section{$\underline{\text { 4.Modélisation des altérations rhéologiques à l'interface }}$}

Si la déformation critique est atteinte, à un niveau $z_{\text {liq }}$ donné par l'équation (11), les paramètres rhéologiques diminuent de quelques ordres de grandeur et le matériau se liquéfie. Ces deux facteurs se traduisent par l'apparition d'une couche limite visqueuse très fine. L'écoulement entre l'interface et la surface de liquéfaction est alors caractérisé par un mouvement de masse homogène (vitesses horizontales uniformes sur la verticale) qui glisse sur un fond lubrifié dû à liquéfaction (Silva Jacinto et Le Hir, 2001). Nous pouvons donc limiter notre analyse à la partie irrotationnelle du mouvement, la partie rotationnelle se "concentrant" dans la surface de liquéfaction. Le domaine d'analyse est schématisé sur la base de la figure 3. Les données du problème sont l'amplitude de la surface libre $\eta_{0}$, la hauteur d'eau h, l'épaisseur de la couche sédimentaire d, la densité de l'eau $\rho_{0}$ et, en fonction de la profondeur, la densité $\rho$ du sédiment.

Les inconnues du problème sont les vitesses et les contraintes dans l'eau et dans le sédiment, l'amplitude d'oscillation $\xi_{0}$ de l'interface et l'état rhéologique près de l'interface $\left(\mathrm{G}_{0}\right.$ et $\delta$ ).

Pour des ondes sinusoïdales dans le temps avec une fréquence $\omega$ et sur l'horizontale avec un nombre d'onde $\mathrm{k}\left(\mathrm{k}=\mathrm{k}_{\mathrm{r}}+\mathrm{i} . \mathrm{k}_{\mathrm{i}}\right)$, toute variable $\mathrm{V}$ peut s'écrire :

$$
V(x, z, t)=V_{0} \cdot e^{i(k x-\omega t)}=\left(V_{0} \cdot e^{-k_{i} x}\right) \cdot e^{i\left(k_{r} x-\omega t\right)}
$$

où $\mathrm{V}_{0}$ donne la distribution verticale de l'amplitude de la variable $\mathrm{V}$. La fréquence est une donnée du problème, alors que le nombre d'onde en est une inconnue. La partie complexe du nombre d'onde $\left(\mathrm{k}_{\mathrm{i}}\right)$ donne naturellement l'échelle de dissipation des ondes.

La théorie linéaire de la houle permet de déduire le mouvement au sein de la colonne d'eau, ainsi que la relation de dispersion des ondes. L'amplitude de pression dans l'eau à l'interface vaut :

$$
\mathrm{p}_{0}=\rho_{0} \mathrm{~g} \eta_{0} / \cosh (\mathrm{kh})
$$

La relation de dispersion donnée par la théorie linéaire en présence d'une oscillation du fond s'écrit :

$$
\omega^{2}=\text { gk.tanh}(\mathrm{kh}) /(1-\varepsilon), \varepsilon=\xi_{0} / \eta_{0} \cdot \cosh (\mathrm{kh})
$$

Au sein de la couche sédimentaire, la vitesse horizontale est uniforme sur la verticale. L'intégration de l'équation de la continuité sur l'épaisseur de la couche sédimentaire et les conditions cinématiques au fond $\left(\mathrm{w}_{0}=0\right)$ et à l'interface $\left(\mathrm{w}_{0}=-\mathrm{i} \omega \xi_{0}\right)$ permettent d'y déterminer les amplitudes des vitesses :

$$
\begin{aligned}
& \mathrm{u}_{0}=\frac{\omega}{\mathrm{k}} \frac{\xi_{0}}{\mathrm{~d}} \\
& \mathrm{w}_{0}=-\mathrm{i} \omega \xi_{0} \frac{\mathrm{z}}{\mathrm{d}}
\end{aligned}
$$

La condition dynamique à l'interface y impose la continuité des contraintes normales. La pression dans l'eau près de l'interface $\left(\mathrm{p}_{0}\right)$ doit équilibrer la contrainte normale dans la couche sédimentaire au niveau de l'interface : la pression $\left(\mathrm{p}_{\mathrm{d}}\right)$ et la contrainte viscoélastique. La condition dynamique linéarisée au voisinage de la position moyenne de l'interface permet d'écrire : 
$\mathrm{p}_{0}-\rho_{0} \mathrm{~g} \xi_{0}=\mathrm{p}_{\mathrm{d}}-\rho \mathrm{g} \xi_{0}-2 \mathrm{G} \frac{\xi_{0}}{\mathrm{~d}} \mathrm{à} \mathrm{z}_{\mathrm{s}}=\mathrm{d}$ et $\mathrm{z}_{\mathrm{e}}=0$

Près de l'interface, la conservation de la quantité de mouvement horizontal s'écrit :

$\rho \frac{\partial \mathrm{u}}{\partial \mathrm{t}}=-\frac{\partial \mathrm{p}_{\mathrm{d}}}{\partial \mathrm{x}}+\rho \mathrm{v} \frac{\partial^{2} \mathrm{u}}{\partial \mathrm{x}^{2}} \Rightarrow \rho \frac{\omega^{2}}{\mathrm{k}^{2}} \frac{\xi_{0}}{\mathrm{~d}}=\mathrm{p}_{\mathrm{d}}+\mathrm{G} \frac{\xi_{0}}{\mathrm{~d}}$

Des équations (17) et (18) peut ainsi être déduite une équation pour l'amplitude de l'interface :

$$
\left[\rho c^{2}-\left(\rho-\rho_{0}\right) g d-3 G\right] \frac{\xi_{0}}{d}=p_{0}
$$

où la célérité c des ondes est donnée par la relation de dispersion (14) et l'amplitude de la pression à l'interface par la théorie linéaire (équation 13).

L'équation (19) permet d'estimer l'oscillation de l'interface engendrée par les oscillations de pression à l'interface. Ces oscillations sont atténuées par les effets stabilisateurs du poids immergé de la couche sédimentaire et de la cohésion. Quand les effets stabilisateurs, dont la cohésion, sont dominants devant l'accélération inertielle, l'interface oscille en opposition de phase vis à vis de la pression à l'interface et donc la surface libre ; l'amplitude d'oscillation est déterminé par la cohésion. Lorsque la célérité des ondes est très élevée et que l'inertie domine, les oscillation sont en phase avec l'oscillation de pression. L'amplitude de l'interface devient indépendante de la cohésion. En situation intermédiaire, quand l'effet inertiel est exactement compensé par les effets stabilisateurs, il y a un effet de résonance : l'amplitude de l'interface augmente, la cohésion diminue et la vase se liquéfie.

L'équation (19) présente deux sources de non-linéarité. Premièrement, la célérité des ondes et donc la relation de dispersion dépendent de l'oscillation générée. Deuxièmement, la cohésion du sédiment dépend de l'amplitude de la déformation induite et donc, aussi, de l'amplitude de l'interface $\left(\left|\gamma_{0}\right|=2\left|\xi_{0} / \mathrm{d}\right|\right)$. La non-linéarité impose une résolution par itération.

Supposant une amplitude de départ (e.g. $\left.\xi_{0}=0\right)$, la célérité et le module de cisaillement complexe sont calculés. Puis, avec ceux-ci, une nouvelle amplitude d'oscillation est estimée. La procédure est poursuivie jusqu'à que la valeur converge.

\section{Résultats}

Prenons comme exemple d'application une situation en eau peu profonde ( $\mathrm{h}=1 \mathrm{~m}, \mathrm{~d}=0.1$ $\mathrm{m})$. Sur la figure $4 \mathrm{a}$, l'effet de l'amplitude des vagues sur l'indice de liquéfaction $(2 \delta / \pi)$ est illustré pour trois densités différentes. Premièrement, nous pouvons retenir un effet de seuil. Il faut une certaine amplitude de la surface libre pour que les contraintes induites au sein du sédiment génèrent la surface de rupture. Cette amplitude de seuil dépend de la rigidité de la couche sédimentaire et donc de sa densité. Une fois l'amplitude seuil dépassée, l'indice de liquéfaction varie, en augmentant, avec l'amplitude de la surface libre. Cette évolution est très proche pour toutes les densités car l'amplitude de l'interface et donc la déformation deviennent presque indépendantes de la densité. En eau peu profonde $(\mathrm{kh}<<1)$ et pour une couche sédimentaire très fine par rapport à la hauteur d'eau $(\mathrm{d} / \mathrm{h}<<1)$, dès que l'effet stabilisateur de la cohésion devient négligeable, l'équation (19) se simplifie et l'amplitude de l'interface devient proportionnelle à celle de la surface libre, légèrement modulée par le rapport des densités :

$$
\frac{\xi_{0}}{\mathrm{~d}}=\frac{\rho_{0}}{\rho} \frac{\mathrm{k}}{\sinh (\mathrm{kh})} \eta_{0} \approx \frac{\rho_{0}}{\rho} \frac{\eta_{0}}{\mathrm{~h}} \text { si kh }<<1
$$



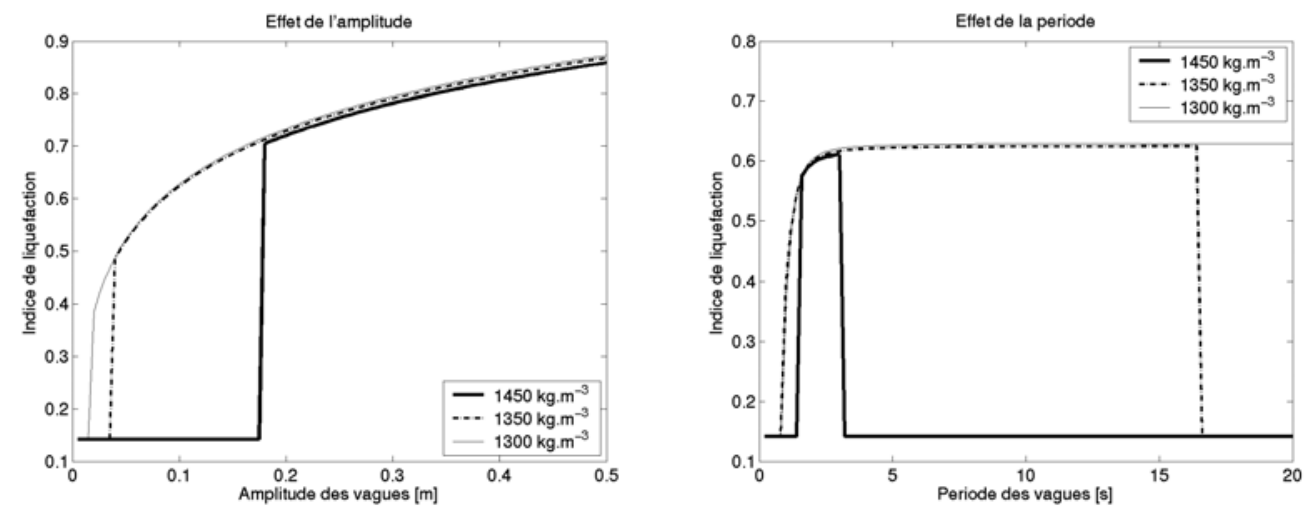

Figure 4 - Indice de liquéfaction en fonction de l'amplitude des vagues et de leur période. Valeurs par défaut : $h=1 \mathrm{~m}, d=0.1 \mathrm{~m}, \omega=1, \eta_{0}=0.1$.

L'effet de la période est illustré sur la figure 4b. L'effet de seuil en fonction de la période s'exprime de deux façons. Pour des périodes très longues, les gradients de pression sont faibles et la mobilisation de la couche sédimentaire devient impossible (équation 10). Pour de périodes très courtes, l'approximation eau peu profonde n'est plus valide et l'amplitude de la pression diminue dans la colonne d'eau, devenant faible à l'interface (équation 13). L'effet d'affaiblissement et de liquéfaction n'est donc possible que sur une gamme de périodes, d'une part, suffisamment importantes pour que les oscillations atteignent considérablement le fond et, d'autre part, suffisamment courtes afin que les gradients horizontaux de pression ne deviennent trop faibles pour mobiliser la couche sédimentaire. Ces deux limites, ou seuils, dépendent de la cohésion initiale du sédiment et donc de sa densité. Par contre, dans la gamme des périodes où la couche sédimentaire est mobilisée, la perte de cohésion se traduit par une oscillation de l'interface et donc un état rhéologique relativement indépendant de la densité : l'oscillation se traduit par un équilibre entre le terme inertiel et l'amplitude de pression à l'interface (équation 20).

\section{$\underline{\text { 6.Conclusions }}$}

Le modèle présenté permet d'analyser l'interaction entre les vagues et les fonds vaseux. Il résulte d'une synthèse de plusieurs observations en laboratoire et in situ ainsi que des résultats de quelques modèles théoriques plus ou moins complexes. Ces travaux concernent d'une part le comportement rhéologique complexe des vases naturelles et, d'autre part, l'interaction houle-vase et les effets de liquéfaction.

Pour des conditions d'agitations données (fréquence, amplitude), en fonction de la profondeur et du profil des caractéristiques rhéologiques au sein du sédiment, cette approche permet d'estimer la profondeur de rupture et l'épaisseur de la couche sédimentaire mobilisable. Puis, il est possible d'estimer l'amplitude d'oscillation de l'interface et d'en déduire la déformation du sédiment qui, de pair avec la densité, détermine les propriétés rhéologiques et l'état de liquéfaction, dont l'affaiblissement $(\phi)$ du module de cisaillement complexe près de l'interface.

Enfin, la réponse dynamique de la couche sédimentaire permet de déterminer la relation de dispersion des ondes, donc leur échelle de dissipation. Le processus de liquéfaction ou d'affaiblissement de la couche sédimentaire est associée à des gammes d'ondes. Les ondes très longues n'engendreront pas de gradients de pressions suffisants. Les ondes très courtes n'atteindront naturellement pas le fond. Mais, dès que la couche sédimentaire est mobilisée, la cohésion au sein du sédiment devient faible devant l'oscillation inertielle. 
La perte de cohésion sous l'effet des vagues augmente naturellement l'érodabilité du sédiment. Dans les formules empiriques d'érosion (e.g. Parheniades, 1977) la rigidité du sédiment est paramétrée par une contrainte critique d'érosion $\left(\tau_{\mathrm{s}}\right)$. Cette contrainte critique d'érosion est proportionnelle à la rigidité initial de sédiment. La contrainte critique d'érosion peut être modulée par l'affaiblissement relatif de la cohésion du sédiment $(\phi)$ sous l'effet des vagues.

Dans les situations où la couche sédimentaire est complètement liquéfiée, le flux à l'interface devient de l'entraînement. Plusieurs auteurs ont proposé des formulations pour l'estimation du flux d'entraînement d'une couche de vase fluide dont, entre autres, Kranenburg et Winterwerp (1997).

\section{Références}

1.Aubry, T., Tolotrahasiina R., Silva Jacinto, R., Bassoullet P. Rheological properties of a natural estuarine mud. Applied Rheology, 13, 142-149, 2003.

Chou, H.-T., M.A. Foda et J.R. Hunt, 1993. Rheological response of cohesive sediments to oscillatory forcing, Coastal and Estuarine Studies, Vol. 42, Nearshore and Estuarine Cohesive Transport, edite by A.J. Mehta, pp. 126-147.

2.Dalrymple, R.A. et P.L. Liu, 1978. Waves over soft bottoms: a two-layer fluid model, Journal of Physical Oceanography, Vol. 8, pp. 1121-1131.

De Wit, P.J. Liquefaction of cohesive sediments caused by waves. Ph. D. Dissertation, Delf University of Technology, 1995.

3.Feng J. Laboratory experiments on cohesive soil-bed fluidization by water waves, Ph.D. Dissertation, Report no. UFL/COEL-92/005, University of Florida, Gainesville, U.S.A, 1992.

4.Foda, M.A., J.R. Hunt et H.-T. Chou, 1993. A nonlinear model for the fluidization of marine mud by waves, Journal of Geophysical Research, vol 98, No C4, pp 7039-7047.

5.Foda, M.A. et S.-Y. Tzang. Resonant fluidization of silty soil by water waves, Journal of Geophysical Research, vol. 99, no. C10, pp 20,463-20,475, 1994.

6.Gade, H.G. Effect of a nonrigid, impermeable bottom on plane surface waves in shallow water, Journal of Marine Research, vol. 16, No. 2, pp.61-82, 1958.

7.Kranenburg, C. et J.C. Winterwerp. Erosion of fluid med layers - I: Entrainment model, ASCE, Journal of Hydraulical Engineering, vol 123, n 6, pp504-511, 1997.

8.Lindenberg, J., L.C. Van Rijn et J.C. Winterwerp. Some experiments on wave induced liquefaction of soft cohesive soil, Journal of Coastal Research, Special Issue No. 5, High Concentration Cohesive Sediment Transport, edited by A.J. Mehta and E.J. Hayter, pp. 127137, 1989.

9.Maa, P.-Y. et A.J. Mehta, 1988. Soft mud properties: voigt model, Journal of Waterway, Port, Coastal and Ocean Engineering, vol 114, No 6, pp 765-770.

10.MacPherson, H., 1980. The attenuation of water waves over a non-rigid bed, Journal of Fluid Mechanics, vol 97, part 4, pp 721-742.

11.Mallard, W.W. et R.A. Dalrymple, 1977. Water waves propagating over a deformable bottom, Proceedings of the 9th Annual Offshore Technology Conference, Vol. 3, pp. 141-146.

12.Mei, C.C. et K.F. Liu, 1987. A Bingham plastic model for a muddy seabed under long waves, Journal of Geophysical Research, Vol. 92, No. C13, pp.14581-14594.

13.Ng Co. Waves over a muddy bed: a two layer Stoke's boundary layer model. Coastal Engineering, 40, pp. 221-242, 2000.

14.Partheniades, E., 1977. Unified view of wash load and bed material load, Journal of Hydraulical Division, 103(9), 1037-1057. 
15.Silva Jacinto, R. et P. Le Hir. Response of stratified muddy beds to water waves. Coastal and Estuarine Fine Sediment Processes, W.H. McAnally and A.J. Mehta (Editors), 2001 Elsevier Science, pp. 95-108, 2001.

16.Yamamoto T., T. Nagai et J.L. Figueroa. Experiments on wave-soil interaction and wave-driven soil transport in clay beds, Continental Shelf Research, vol. 5, pp 521-540, 1986. 